\title{
Supersymmetry Signatures with Tau Jets at the Tevatron
}

\author{
Joseph D. Lykken and Konstantin T. Matchev \\ Theoretical Physics Department, Fermi National Accelerator Laboratory, Batavia, IL 60510, USA
}

\begin{abstract}
We study the supersymmetry reach of the Tevatron in channels containing both isolated leptons and identified tau jets. In the most challenging case, where the branching ratios of gauginos to taus dominate, we find that searches for two leptons, a tau jet and a large amount of missing transverse energy have a much better reach than the classic trilepton signature. With total integrated luminosity of $\mathrm{L} \gtrsim 4 \mathrm{fb}^{-1}$, the Tevatron will start extending the expected LEP-II reach for supersymmetry.
\end{abstract}

PACS numbers: 12.60.Jv, 14.80.Ly, 13.35.Dx

FERMILAB-PUB-99/034-T

Searches for supersymmetry (SUSY) in Run I of the Tevatron have been done exclusively in channels involving some combination of leptons, jets, photons and missing transverse energy $\left(E_{T}\right)$ [1]. At the same time, several Run I analyses have identified hadronic tau jets, e.g. in $W$-production [2] and top decays [3]. Hadronic taus have also been used to place limits on a charged Higgs [- 1 ] and leptoquarks [5]. As tau identification is expected to improve further in Run II, this raises the question whether SUSY searches in channels involving tau jets are feasible.

SUSY signatures with tau leptons are very well motivated, since they arise in a variety of models of low-energy supersymmetry, e.g. gravity-mediated [6 8] or the minimal gauge-mediated models [8 10]. In this paper we shall study all possible experimental signatures with three identified objects (leptons or tau jets) plus $\not_{T}$, and compare their reach to the clean trilepton channel [11,12], which is the classic SUSY signature at the Tevatron. It arises in the decays of gaugino-like chargino-neutralino pairs $\tilde{\chi}_{1}^{ \pm} \tilde{\chi}_{2}^{0}$. The reach is somewhat limited by their rather small leptonic branching fractions. In the limit of either heavy or equal in mass squarks and sleptons, the leptonic branching ratios of $\tilde{\chi}_{1}^{ \pm}$and $\tilde{\chi}_{2}^{0}$ are $W$-like and $Z$-like, respectively. However, both gravity-mediated and gauge-mediated models of SUSY breaking allow the sleptons to be much lighter than the squarks, thus enhancing the leptonic branching fractions of the gauginos.

There are at least three generic reasons as to why one may expect light sleptons in the spectrum. First, the slepton masses at the high-energy scale may be rather small to begin with. This is typical for gauge-mediated models, since the sleptons are colorless and do not receive large soft mass contributions proportional to the strong coupling constant $\alpha_{s}$. The minimal gravity-mediated (mSUGRA) models, on the other hand, predict light sleptons if the universal scalar mass $M_{0}$ is much smaller than the universal gaugino mass $M_{1 / 2}$. Second, the renormalization group equations for the scalar soft masses contain terms proportional to Yukawa couplings, which tend to reduce the corresponding mass during the evolution down to low-energy scales. This effect is significant for third generation scalars, and for large values of $\tan \beta$ (the ratio of the Higgs vacuum expectation values $v_{2}$ and $v_{1}$ ) splits the staus from the first two generation sleptons. And finally, the mixing in the charged slepton mass matrix further reduces the mass of the lightest eigenstate. The slepton mixing is enhanced at large $\tan \beta$, since it is proportional to $\mu m_{l} \tan \beta / m_{\tilde{l}}^{2}$, where $m_{l}$ $\left(m_{\tilde{l}}\right)$ is the lepton (slepton) mass and $\mu$ is the supersymmetric Higgs mass parameter. Notice that this effect again only applies to the staus, since $m_{\tau} \gg m_{\mu, e}$.

Due to these three effects, it may very well be that among all scalars, only the lightest sleptons from each generation (or just the lightest stau $\tilde{\tau}_{1}$ ) are lighter than $\tilde{\chi}_{1}^{ \pm}$and $\tilde{\chi}_{2}^{0}$. Indeed, in both gravity-mediated and gauge-mediated models one readily finds regions of parameter space where either $m_{\tilde{\chi}_{1}^{0}}<m_{\tilde{\tau}_{1}} \sim m_{\tilde{\mu}_{R}}<m_{\tilde{\chi}_{1}^{+}} \sim m_{\tilde{\chi}_{2}^{0}}$ (typically at small $\tan \beta$ ) or $m_{\tilde{\chi}_{1}^{0}}<m_{\tilde{\tau}_{1}}<m_{\tilde{\chi}_{1}^{+}} \sim m_{\tilde{\chi}_{2}^{0}}<m_{\tilde{\mu}_{R}}$ (at large $\tan \beta$ ). Depending on the particular model, and the values of the parameters, the gaugino pair decay chain may then end up overwhelmingly in any one of the four final states: $l l l, l l \tau$, $l \tau \tau$ or $\tau \tau \tau$.

In order to make a final decision as to which experimental signatures are most promising, we have to factor in the tau branching ratios to leptons and jets. About two-thirds of the subsequent tau decays are hadronic, so it appears advantageous to consider signatures with tau jets in the final state as alternatives to the clean trilepton signal. (From now on, we shall use the following terminology: a "lepton" $(l)$ is either a muon or an electron; a tau is a tau-lepton, which can later decay either leptonically, or to a hadronic tau jet, which we denote by $\tau_{h}$.) The presence of taus in the underlying SUSY signal always leads to an enhancement of the signatures with tau jets in comparison to the clean trileptons. This disparity is most striking for the case of $\tau \tau \tau$ decays, where $B R\left(\tau \tau \tau \rightarrow l l \tau_{h}\right) / B R(\tau \tau \tau \rightarrow l l l) \sim 5.5$. An additional advantage of the tau jet channels is that the leptons from tau decays are much softer than the tau jets and as a result will have a relatively low reconstruction efficiency.

On the other hand, the tau jet channels suffer from larger backgrounds than the clean trileptons. The physical background (from real tau jets in the event) is actually smaller, but a significant part of the background is due to 
events containing narrow isolated QCD jets with the correct track multiplicity, which can be misidentified as taus. The jetty signatures are also hurt by the lower detector efficiency for tau jets than for leptons. The main goal of our study, therefore, will be to see what is the net effect of all these factors, on a channel by channel basis.

For our analysis we choose to examine one of the most challenging scenarios for SUSY discovery at the Tevatron. We shall assume the typical large $\tan \beta$ mass hierarchy $m_{\tilde{\chi}_{1}^{0}}<m_{\tilde{\tau}_{1}}<m_{\tilde{\chi}_{1}^{+}}<m_{\tilde{\mu}_{R}}$. One then finds that $B R\left(\tilde{\chi}_{1}^{+} \tilde{\chi}_{2}^{0} \rightarrow\right.$ $\tau \tau \tau+X) \simeq 100 \%$ below $\tilde{\chi}_{1}^{ \pm} \rightarrow W^{ \pm} \tilde{\chi}_{1}^{0}$ and $\tilde{\chi}_{2}^{0} \rightarrow Z \tilde{\chi}_{1}^{0}$ thresholds. In order to shy away from specific model dependence, we shall conservatively ignore all SUSY production channels other than $\tilde{\chi}_{1}^{ \pm} \tilde{\chi}_{2}^{0}$ pair production. The $p_{T}$ spectrum of the taus resulting from the chargino and neutralino decays depends on the mass differences $m_{\tilde{\chi}_{1}^{+}}-m_{\tilde{\tau}_{1}}$ and $m_{\tilde{\tau}_{1}}-m_{\tilde{\chi}_{1}^{0}}$. The larger they are, the harder the spectrum, and the better the detector efficiency. However, as the mass difference gets large, the $\tilde{\chi}_{1}^{+}$and $\tilde{\chi}_{2}^{0}$ masses themselves become large too, so the production crosssection is severely suppressed. Therefore, at the Tevatron we can only explore regions with favorable mass ratios and at the same time small enough gaugino masses. This suggests a choice of SUSY mass ratios: for definiteness we fix $2 m_{\tilde{\chi}_{1}^{0}} \sim(4 / 3) m_{\tilde{\tau}_{1}} \sim m_{\tilde{\chi}_{1}^{+}}\left(<m_{\tilde{\mu}_{R}}\right)$ throughout the analysis, and vary the chargino mass. The rest of the superpartners have fixed large masses corresponding to the mSUGRA point $M_{0}=180 \mathrm{GeV}, M_{1 / 2}=180 \mathrm{GeV}, A_{0}=0$ $\mathrm{GeV}, \tan \beta=44$ and $\mu>0$, but we are not constrained to mSUGRA models only. Our analysis will apply equally to gauge-mediated models with a long-lived neutralino NLSP, as long as the relevant gaugino and slepton mass relations are similar. Note that our choice of heavy first two generation sleptons is very conservative. A more judicious choice of their masses, namely $m_{\tilde{\mu}_{R}}<m_{\tilde{\chi}_{1}^{+}}$, would lead to a larger fraction of trilepton events, and as a result, a higher reach. Furthermore, the gauginos would then decay via two-body modes to first generation sleptons, and the resulting lepton spectrum would be much harder, leading to a higher lepton efficiency. Notice also that the $\tilde{\chi}_{1}^{ \pm} \tilde{\chi}_{2}^{0}$ production cross-section is sensitive to the squark masses, but since this is the only production process we are considering, our results can be trivially rescaled to account for a different choice of squark masses, or to include other production processes as well.

Since the experimental signatures in our analysis contain only soft leptons and tau jets, an important issue is whether one can develop efficient combinations of Level 1 and Level 2 triggers to accumulate these data sets without squandering all of the available bandwidth. We will not attempt to address this issue here; instead we will assume $100 \%$ trigger efficiency for those signal events which pass all of our analysis and acceptance cuts. We have nevertheless studied the following set of triggers: 1$) E_{T}>40 \mathrm{GeV}$; 2$) p_{T}(l)>20 \mathrm{GeV}$ and 3$) p_{T}(l)>10 \mathrm{GeV}, p_{T}($ jet$)>15 \mathrm{GeV}$ and $E_{T}>15 \mathrm{GeV}$; with pseudorapidity cuts $|\eta(e)|<2.0,|\eta(\mu)|<1.5$ and $|\eta(j e t)|<4.0$. We found that they are efficient in picking out about $90 \%$ of the signal events in the channels with at least one lepton (see below). Dedicated low $p_{T}$ tau triggers for Run II, which may be suitable for the new tau jet channels, are now being considered by both CDF 13] and D0 14.

We used PYTHIA v6.115 and TAUOLA v2.5 for event generation. We used the SHW v2.2 package [15], which simulates an average of the CDF and D0 Run II detector performance. In SHW tau objects are defined as jets with $|\eta|<1.5$, net charge \pm 1 , one or three tracks in a $10^{\circ}$ cone with no additional tracks in a $30^{\circ}$ cone, $E_{T}>5 \mathrm{GeV}$, $p_{T}>5 \mathrm{GeV}$, plus an electron rejection cut. SHW electrons are required to have $|\eta|<1.5, E_{T}>5 \mathrm{GeV}$, hadronic to electromagnetic energy deposit ratio $R_{h / e}<0.125$, and satisfy standard isolation cuts. Muon objects are required to have $|\eta|<1.5, E_{T}>3 \mathrm{GeV}$ and are reconstructed using Run I efficiencies. We use standard isolation cuts for muons as well. Jets are required to have $|\eta|<4, E_{T}>15 \mathrm{GeV}$. In addition we have added jet energy correction for muons and the rather loose id requirement $R_{h / e}>0.1$. We have also modified the TAUOLA program in order to correctly account for the chirality of tau leptons coming from SUSY decays.

The reconstruction algorithms in SHW already include some basic cuts, so we can define a reconstruction efficiency $\epsilon_{\text {rec }}$ for the various types of objects: electrons, muons, tau jets etc. We find that as we vary the chargino mass from 100 to $140 \mathrm{GeV}$ the lepton and tau jet reconstruction efficiencies for the signal range from 42 to $49 \%$, and from 29 to $36 \%$, correspondingly. The lepton efficiency may seem surprisingly low, but this is because a lot of the leptons are very soft and fail the $E_{T}$ cut. The tau efficiency is in good agreement with the results from Ref. [16] and [17], once we account for the different environment, as well as cuts used in those analyses.

The most important background issue in the new tau channels is the fake tau rate. Several experimental analyses try to estimate it using Run I data. Here we simulate the corresponding backgrounds to our signal and use SHW to obtain the fake rate, thus avoiding trigger bias [16]. We find that the tau fake rate in $W$ production is $1.5 \%$, independent of the tau $p_{T}$, which is in agreement with the findings of Refs. 16 18].

In the following we list our cuts for each channel.

In order to maximize the reach in the $l l l E_{T}$ channel, we apply the soft lepton $p_{T}$ cuts advertised in Refs. 11]. We require a central lepton with $p_{T}>11 \mathrm{GeV}$ and $|\eta|<1.0$, and in addition two more leptons with $p_{T}>7 \mathrm{GeV}$ and $p_{T}>5 \mathrm{GeV}$. Leptons have to be isolated: $I(l)<2 \mathrm{GeV}$, where $I$ is the total transverse energy contained in a cone of 
size $\delta R=\sqrt{\Delta \varphi^{2}+\Delta \eta^{2}}=0.4$ around the lepton. We impose a dilepton invariant mass cut for same flavor, opposite sign leptons: $\left|m_{l l}-M_{Z}\right|>10 \mathrm{GeV}$ and $\left|m_{l l}\right|>11$. Finally, we impose an optional veto on additional jets and require $E_{T}$ to be either more than $20 \mathrm{GeV}$, or $25 \mathrm{GeV}$. This gives us a total of four combinations of the $E_{T}$ cut and the jet veto $(\mathrm{JV})\left(\mathrm{A}: E_{T}>20 \mathrm{GeV}\right.$, no JV; B: $E_{T}>25 \mathrm{GeV}$, no JV; C: $E_{T}>20 \mathrm{GeV}$, with JV; D: $E_{T}>25 \mathrm{GeV}$, with JV), which we apply for all tau jet signatures later as well.

For our $l l \tau_{h} E_{T}$ analysis we impose cuts similar to the stop search analysis in the $l^{+} l^{-} j E_{T}$ channel [19]: two isolated $(I(l)<2 \mathrm{GeV})$ leptons with $p_{T}>8 \mathrm{GeV}$ and $p_{T}>5 \mathrm{GeV}$, and one identified tau jet with $p_{T}\left(\tau_{h}\right)>15 \mathrm{GeV}$. Again, we impose the above invariant mass cuts for any same flavor, opposite sign dilepton pair. This channel was also studied in Ref. [7] with somewhat harder cuts on the leptons. A separate, very interesting signature $\left(l^{+} l^{+} \tau_{h} E_{T}\right)$ arises if the two leptons have the same sign, since the background is greatly suppressed. In fact, we expect this background to be significantly smaller than the trilepton background! Roughly one third of the signal events in the general $l l \tau_{h}$ sample are expected to have like-sign leptons.

For our $l \tau_{h} \tau_{h} E_{T}$ analysis we use some basic identification cuts: two tau jets with $p_{T}>15 \mathrm{GeV}$ and $p_{T}>10 \mathrm{GeV}$ and one isolated lepton with $p_{T}>7 \mathrm{GeV}$.

Finally, for the $\tau_{h} \tau_{h} \tau_{h} E_{T}$ signature we only require three tau jets with $p_{T}>15,10$ and $8 \mathrm{GeV}$, respectively.

One can get a good idea of the relative importance of the different channels by looking at the corresponding signal samples after the analysis cuts have been applied. In Fig. 1 we show the signal cross-sections times the corresponding branching ratios times the total efficiency $\epsilon_{\text {tot }} \equiv \epsilon_{\text {rec }} \epsilon_{c u t s}$, which accounts for both the detector acceptance $\epsilon_{\text {rec }}$ and the efficiency of the cuts $\epsilon_{\text {cuts }}$ (for each signal point we generated $10^{5}$ events). We see that the lines are roughly ordered according to the branching ratios of three taus into the corresponding final state signatures. This can be understood as follows. The acceptance (which includes the basic ID cuts in SHW) is higher for leptons than for $\tau$ jets. Therefore, replacing a lepton with a tau jet in the experimental signature costs us a factor of $\sim 1.5$ in acceptance, due to the poorer reconstruction of tau jets, compared to leptons. Later, however, the cuts tend to reduce the leptonic signal more than the tau jet signal, mostly because the leptons are softer than the tau jets. It turns out that these two effects mostly cancel each other, and the total efficiency $\epsilon_{t o t}$ is roughly the same for all channels. Therefore the relative importance of each channel will only depend on the tau branching ratios and the backgrounds. For example, in going from $l l l$ to $l l \tau_{h}$, one wins a factor of 5.5 from the branching ratio. Therefore the background to $l l \tau_{h} E_{T}$ must be at least $5.5^{2} \sim 30$ times larger in order for the clean trilepton channel to be still preferred.

We next turn to the discussion of the backgrounds involved. We have simulated the following physics background processes: $Z Z, W Z, W W, t \bar{t}, Z+$ jets, and $W+$ jets, generating $4 \times\left(10^{6}\right)$ and $2 \times\left(10^{7}\right)$ events, respectively. We list the results in Table [1], where we show the total background cross-section $\sigma_{B G}$ for each case A-D, as well as the contributions from the individual processes, for case A. All errors are purely statistical. Our simulated background in the trilepton channel is higher than previously found in Refs. [7, 11], which employed ISAJET for event generation. Current versions of ISAJET simulate the $W Z$ and $Z Z$ processes in the limit of zero $Z$ width. We find that most of the $W Z$ and $Z Z$ background events from PYTHIA contain an off-shell $Z$ instead and pass the dilepton invariant mass cuts. Unfortunately, neither ISAJET, nor PYTHIA contain the $W \gamma$ interference contribution to $W Z$, so our result still somewhat underestimates the $W Z$ trilepton background. As we move to the channels with tau jets, the number of events with real tau jets decreases, mostly because of the smaller branching ratios of $W$ and $Z$ to taus. However, the contribution from events with fake tau jets increases, and for the $3 \tau_{h}$ channel events with fakes are the dominant part of the background. Our results for the $l l \tau_{h}$ and $l \tau_{h} \tau_{h}$ channels differ from those of Ref. [10, which assumed a $p_{T}$-dependent fake tau rate of only $0.1-0.5 \%$.

We find that although the jet veto successfully removes the $t \bar{t}$ background to the first three channels, it also reduces the signal (see Fig. 1), and hence does not improve signal-to-noise. A higher $E_{T}$ cut also never seemed to help. Indeed, the major backgrounds contain leptonic $t$ and $W$ decays and tend to have a lot of missing energy. Notice that we did not account for fake leptons in our $Z+$ jets backgrounds to $3 l$ and $l^{+} l^{+} \tau_{h}$, since SHW does not provide a realistic simulation of those. The best way to estimate this background component will be from Run II data (an analysis based on Run I data [20] reveals that the $3 l$ contribution from fakes is comparable to our result in Table [] which only includes real isolated leptons from heavy flavor jets). We have a bit underestimated the total background to the $3 \tau_{h}$ channel by considering only processes with at least one real tau in the event. We expect sizable contributions from pure QCD multijet events, or $W j \rightarrow j j j$, where all three tau jets are fake.

A $3 \sigma$ exclusion limit requires a total integrated luminosity $L(3 \sigma)=9 \sigma_{B G}\left\{\sigma_{\text {sig }} B R\left(\tilde{\chi}_{1}^{+} \tilde{\chi}_{2}^{0} \rightarrow X\right) \epsilon_{t o t}\right\}^{-2}$. Notice that $L(3 \sigma)$ depends linearly on the background $\sigma_{B G}$ after cuts, but quadratically on the signal branching ratios. This allows the jetty channels to compete very successfully with the clean trilepton signature, whose branching ratio is quite small. In Fig. 2 we show the Tevatron reach in the three best channels: trileptons $(\times)$, dileptons plus a tau jet $(\square)$ and like-sign dileptons plus a tau jet $(\diamond)$. We see that the two channels with tau jets have a much better sensitivity 
compared to the usual trilepton signature. Assuming that efficient triggers can be implemented, the Tevatron reach will start exceeding LEP II limits as soon as Run II is completed and the two collaborations have collected a total of $4 \mathrm{fb}^{-1}$ of data. Considering the intrinsic difficulty of the SUSY scenario we are contemplating, the mass reach for Run III is quite impressive. One should also keep in mind that we did not attempt to optimize our cuts for the new channels. For example, one could use angular correlation cuts to suppress Drell-Yan, transverse $W$ mass cut to suppress $W Z$ [21], or (chargino) mass-dependent $p_{T}$ cuts for the leptons and tau jets, to squeeze out some extra reach. In addition, the $l l \tau_{h}$ channel can be explored at smaller values of $\tan \beta$ as well [7, 11, 20], since the two-body chargino decays are preferentially to tau sleptons. In that case, the clean trilepton channel still offers the best reach, and a signal can be observed already in Run II. Then, the tau channels will not only provide an important confirmation, but also hint towards some probable values of the SUSY model parameters.

Acknowledgements. We would like to thank V. Barger, J. Conway, R. Demina, L. Groer, J. Nachtman, D. Pierce, A. Savoy-Navarro, M. Schmitt and A. Turcott for useful discussions. Fermilab is operated under DOE contract DEAC02-76CH03000.

[1] See, e.g. M. Carena et al., preprint ANL-HEP-PR-97-98.

[2] F. Abe et al., Phys. Rev. Lett. 68, 3398 (1992); A. Kotwal, ICHEP'98 Proceedings, Vancouver, Canada, July 23-29, 1998; S. Protopopescu, preprint FERMILAB-CONF-98-376-E.

[3] M. Hohlmann, preprint FERMILAB-CONF-96-330-E; F. Abe et al., Phys. Rev. Lett. 79, 3585 (1997).

[4] F. Abe et al., Phys. Rev. D54, 735 (1996); Phys. Rev. Lett. 79, 357 (1997).

[5] F. Abe et al., Phys. Rev. Lett. 78, 2906 (1997); preprint FERMILAB-PUB-98-352-E.

[6] H. Baer et al., Phys. Rev. Lett. 79, 986 (1997).

[7] H. Baer et al., Phys. Rev. D58, 075008 (1998).

[8] J. Wells, Mod. Phys. Lett. A13, 1923 (1998).

[9] B. Dutta et al., preprint OSU-HEP-98-4; D. Muller and S. Nandi, preprint OSU-HEP-98-8.

[10] H. Baer et al., preprint FSU-HEP-990305.

[11] V. Barger et al., Phys. Lett. B433, 328 (1998); V. Barger and C. P. Kao, preprint FERMILAB-PUB-98-342-T.

[12] F. Abe et al., preprint FERMILAB-PUB-98/084-E; B. Abbott et al., Phys. Rev. Lett. 80, 1591 (1998).

[13] Y. Seiya and A. Navoy-Savarro, talks given at the SUSY/Higgs Workshop, Fermilab, 1998.

[14] A. Turcott, private communication.

[15] J. Conway, talk given at the SUSY/Higgs Workshop meeting, Fermilab, May 14-16, 1998, additional information available at www.physics.rutgers.edu/jconway/soft/shw/shw.html.

[16] M. Hohlmann, Univ. of Chicago Ph.D. Thesis, 1997.

[17] L. Groer, Rutgers University Ph.D. Thesis, 1998.

[18] E. Smith, talk given at the Higgs and Supersymmetry conference, Gainesville, FL, March 7-11, 1999.

[19] R. Demina, talk given at the SUSY/Higgs Workshop meeting, Fermilab, November 19-21, 1998.

[20] K. Matchev and D. Pierce, preprint FERMILAB-Pub-99/078-T.

[21] H. Baer, M. Drees, F. Paige, P. Quintana and X. Tata, preprint FSU-HEP-990509. 
TABLE I. Total background cross-section after cuts $\sigma_{B G}$ (in $\mathrm{fb}$ ) for the various channels in cases A-D, as well as the breakdown of the individual contributions for case A.

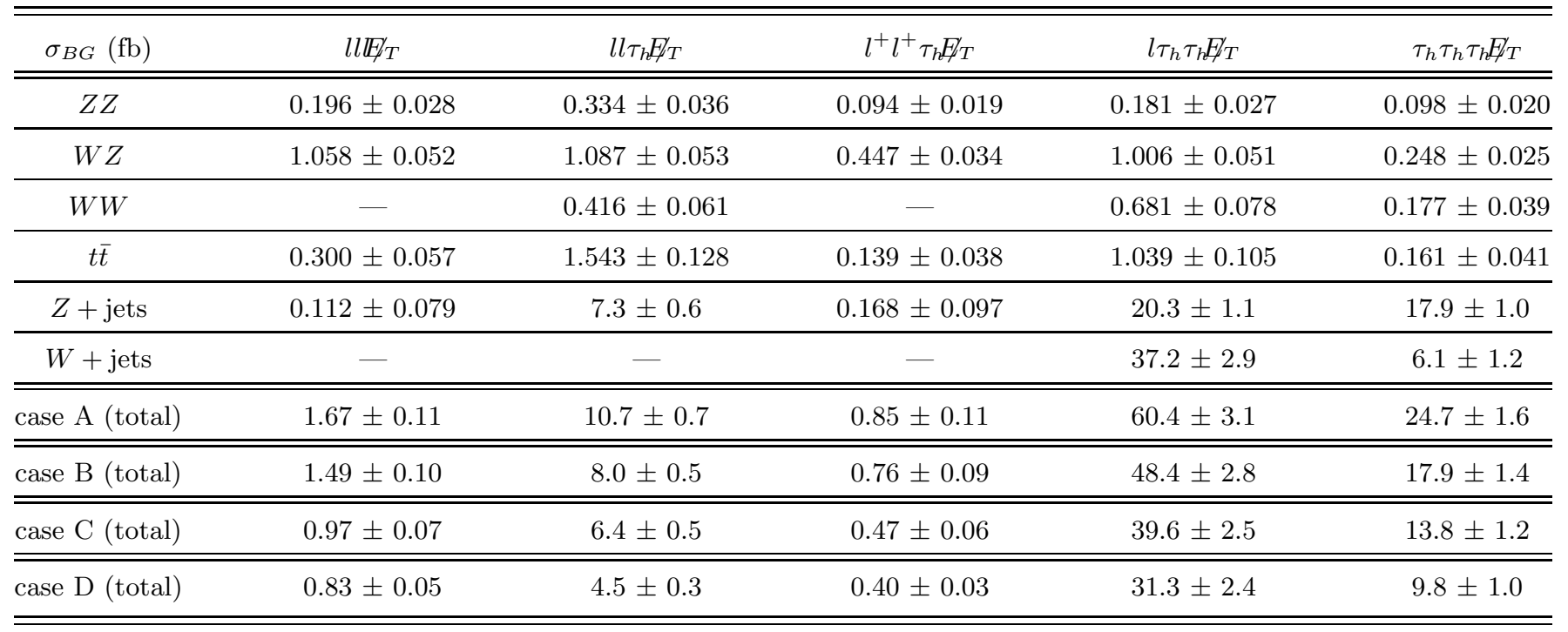




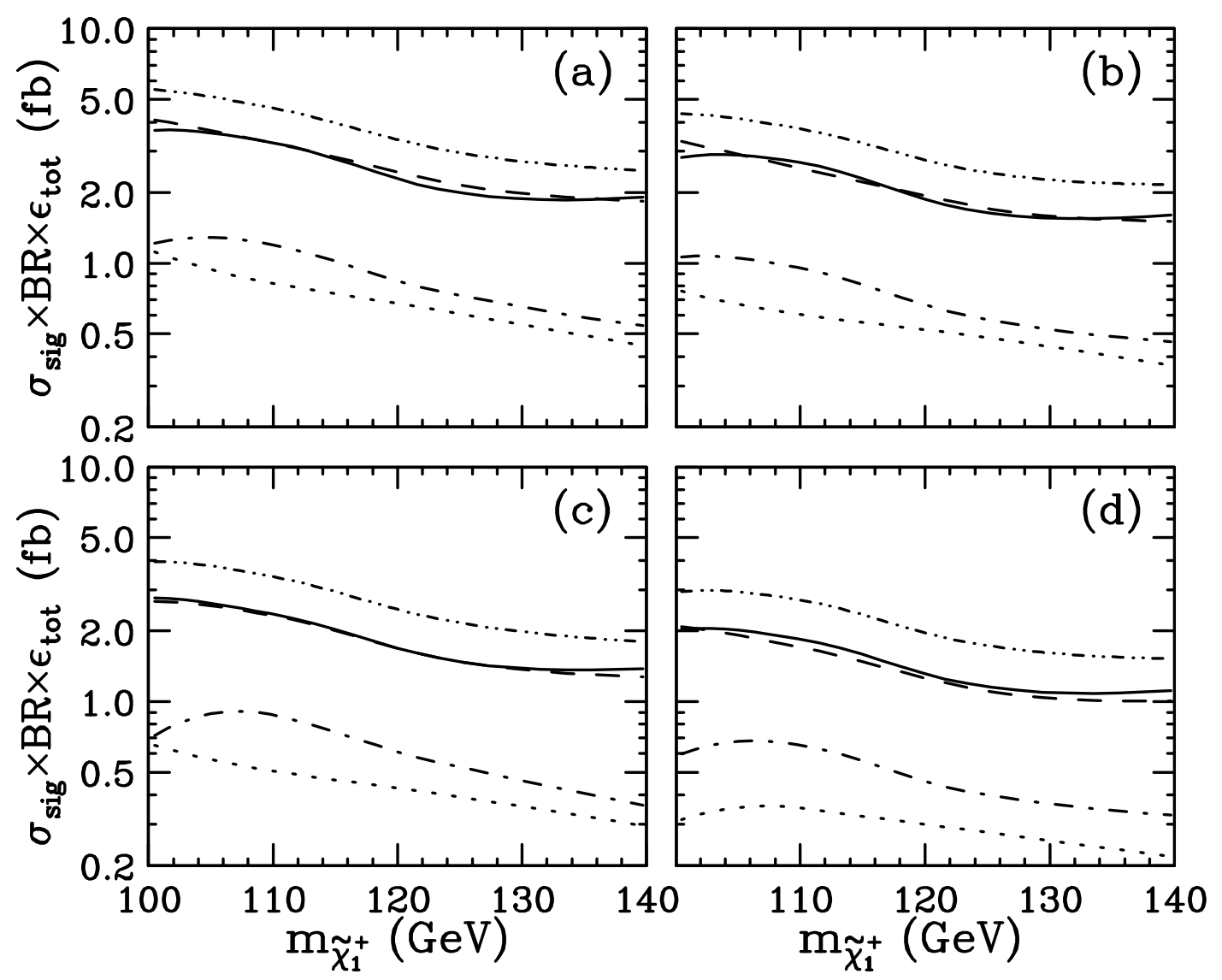

FIG. 1. Signal cross-section times branching ratio after cuts for the five channels discussed in the text: $l l l \mathbb{E}_{T}$ (dotted), $l l \tau_{h} \#_{T}$ (dashed), $l^{+} l^{+} \tau_{h} \#_{T}$ (dot dashed), $l \tau_{h} \tau_{h} \#_{T}$ (dot dot dashed) and $\tau_{h} \tau_{h} \tau_{h} \#_{T}$ (solid); and for various sets of cuts: (a) cuts A, (b) cuts B, (c) cuts C and (d) cuts D. 

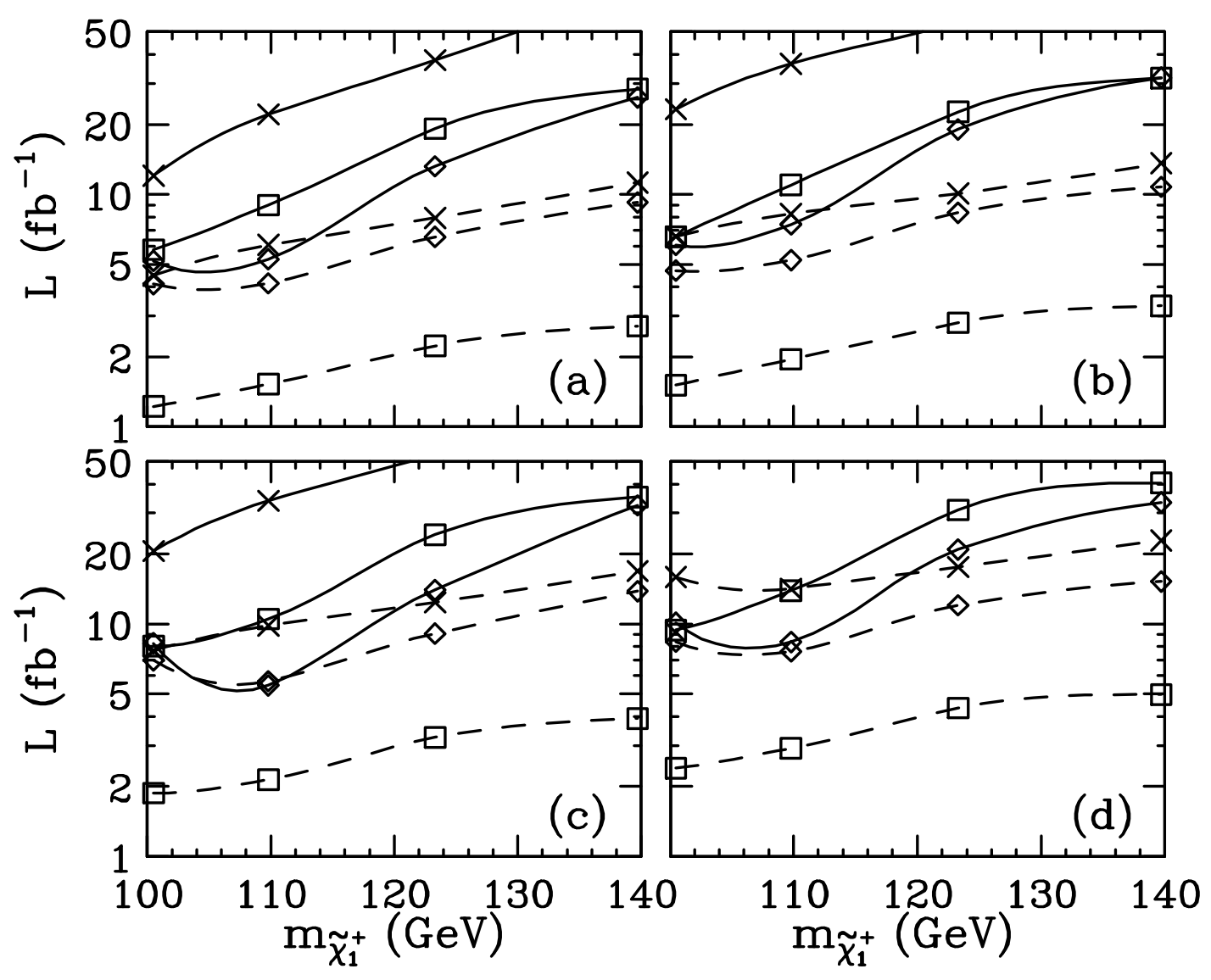

FIG. 2. The total integrated luminosity $L$ needed for a $3 \sigma$ exclusion (solid lines) or observation of 5 signal events (dashed lines), as a function of the chargino mass $m_{\tilde{\chi}_{1}^{+}}$, for the three channels: $l l l \not_{T}(\times), l l \tau_{h} E_{T}(\square)$ and $l^{+} l^{+} \tau_{h} \not_{T}(\diamond)$; and for various sets of cuts: (a) cuts A; (b) cuts B; (c) cuts C and (d) cuts D. 